\title{
A Case of Heterotopic Pregnancy
}

\section{Kaberi Majumder ${ }^{1 *}$ \\ Anisul Moula ${ }^{1}$}

Department of Radiology and Imaging Chattagram Maa-O-Shishu Hospital Medical College Chittagong, Bangladesh.
*Correspondence to:

\section{Dr. Kaberi Majumder} Assistant Professor

Department of Radiology and Imaging Chattagram Maa-O-Shishu Hospital Medical College Chittagong, Bangladesh.

Mobile : +8801817207628

E-mail: drkaberi3r@gmail.com

http://www.banglajol.info/index.php/CMOSHMCJ

\begin{abstract}
We are presenting a case report of heterotopic pregnancy, which is diagnosed during ultrasound examination of a gravid woman, 23 year old, referred from outpatient department. Heterotopic pregnancy is a rare obstetrics phenomenon and carries a significant natural morbidity and mortality due to risk of rupture of the ectopic pregnancy. Clinicians and sonographers may fall into a false sense of security when an intrauterine gestational sac is identified. This results in inadequate inspection of the adnexae and remaining structures during ultrasonography despite a strong initial clinical suspicion of ectopic pregnancy. Hence, a thorough ultrasonographic examination is needed in managing these patients, especially when there is a high suspicion of ectopic pregnancy or in the presence of pelvic free fluid even when an intrauterine pregnancy is identified.
\end{abstract}

Key words: Heterotopic pregnancy; Ectopic pregnancy; Ultrasonography.

\section{INTRODUCTION}

A heterotopic pregnancy is a rare situation when there is an intra-uterine and extrauterine pregnancy occurring simultaneously. In orther words one fetus has implanted outside the uterus (usually in fallopian tube), while others is in the uterus. It may also be referred to as a combined ectopic pregnancy, multiple sited pregnancy or coincidental pregnancy. The estimated incidence in the general population is low and ranges in between 1:400 to 30000 (for a naturally conceived pregnancy ${ }^{1}$. The incidence among patient with assisted reproduction is higher and is approximately 1 in 7000 overall and as high as 1 in 900 with ovulation induction ${ }^{2,3}$. Heterotopic pregnancy have been diagnosed from 05 to 34 weeks of gestation with up to $70 \%$ diagnosed between $05-08$ weeks of gestation, $20 \%$ between $09-10$ weeks and only $10 \%$ after $11^{\text {th }}$ week $^{4}$.

Recognized risk factors predisposing to heterotopic pregnancy include:

- Pelvic adhesive disease

- Effects of Diethylstilbestrol(DES) on the genital tract

- Use of an Intra-Uterine Contraceptive Device( IUCD)

- Previous history of tubal surgery to treat infertility, ectopic pregnancy or tubal adhesion

- History of previous ectopic pregnancy

\section{CASE REPORT}

An woman of 23 years of age hailing from Chittagong sub-urban area, attended Gynaecology and Obstetric outpatient department of Chattagram Maa-O-Shishu General Hospital (CMSOGH) with a history of 09 weeks of amenorrhoea, lower abdominal pain for 01 day, and urinary retention for $8 \mathrm{hrs}$. She denied any per vaginal bleeding and bowel symptoms.

Her menstrual history was irregular and she had history of using Oral Contraceptive Pill (OCP). She had a past history of miscarriage and undergone fertility treatment. Her past medical and surgical histories were unremarkable. 
On examination, she was tachycardic at 105 beats per min with a BP of 130/60 mm Hg. Her abdomen was soft with suprapubic and right iliac fossa tenderness.

After initial resuscitation with IV fluid, she was investigated by urine pregnancy test, which was positive. On trans-abdominal ultrasound, it revealed a $07+$ weeks of nonviable intrauterine pregnancy (Figure 1) and a large complex mass (11.0 cm x 12.0 $\mathrm{cm}$ ) with a gestational sac like structure in right adnexal region (Figure 2). A moderate amount of fluid was present in Cul-desac and in Morrison's space. A diagnosis of heterotopic pregnancy with tubal rupture was made. On emergency laparotomy revealed a $07+$ week's gravid uterus and rupture of the right fallopian tube and presence of haemoperitoneum. Right sided salphingectomy with removal of haemoperitoneum and peritoneal lavage were performed. She was transfused 02 units of blood during and after surgery, and her postoperative period was uneventful.
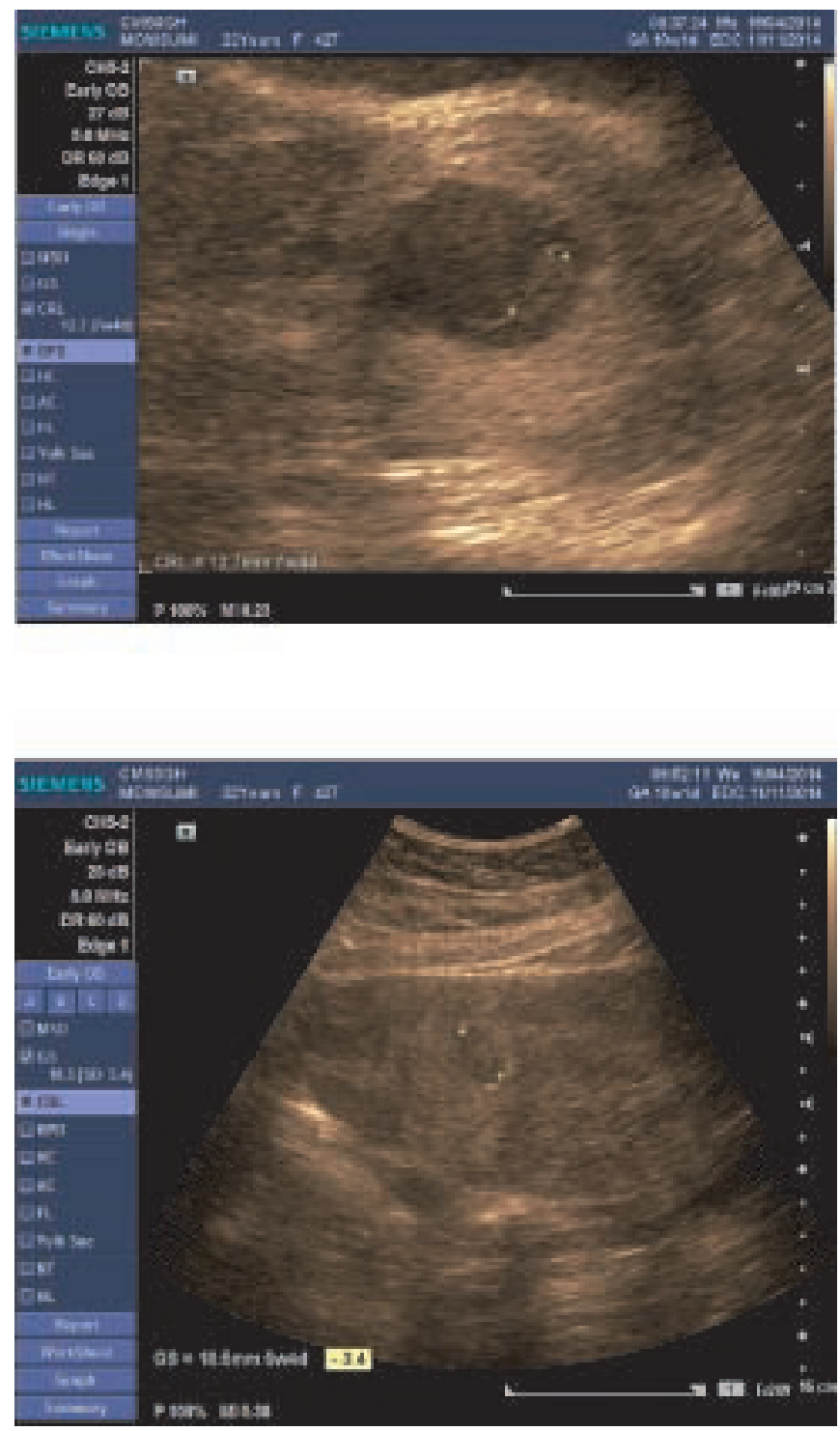

Figure 2 : On trans-abdominal ultrasound, a large complex right adnexal mass with a gestational sac

\section{DISCUSSION}

Heterotopic pregnancy is defined as the presence of multiple gestations, with one being in the uterine cavity and the other outside the uterus, commonly in the fallopian tube and rare in the cervix or ovary ${ }^{5,6}$.

Heterotopic pregnancies are becoming more common following assisted conception techniques for sub fertility ${ }^{7}$. However spontaneous heterotopic pregnancies are quite $\operatorname{rare}^{8}$. The incidence quoted is 1 in 30,000 pregnancies 9 .

Around $50 \%$ of heterotopic pregnancies are asymptomatic ${ }^{10}$. When symptomatic, the main clinical manifestations are abdominal pain due to peritoneal irritation, adnexal mass with or without vaginal bleeding and hypovolumic shock. However, clinical manifestations are more frequent in situations of tubal rupture.

To assist in early screening and diagnosing of ectopic and heterotopic pregnancies, beta-hCG serum assaying, along with vaginal ultrasound, can be routinely used in prenatal examinations during the early stages of pregnancy ${ }^{11}$. A diagnosis of a pseudo-sac should be made with caution, as even in the presence of a pseudo-sac, there can be a high false positive diagnosis of an ectopic pregnancy ${ }^{12}$. Sometimes the presence of a haemorrhagic corpus luteum can confuse and delay in the diagnosis of a heterotopic pregnancy ${ }^{13}$.

In normal pregnancies with blood beta HCG levels above 1500 $2000 \mathrm{mIU} / \mathrm{ml}$, the intrauterine image of pregnancy should already be detectable. However, identifying the intrauterine image does not exclude the possibilities of heterotopic pregnancy, which is more frequent with fertility treatments ${ }^{4,14}$. Thus, adequate viewing of adnexa becomes necessary in all assessments on the start of pregnancy.

With an increase in assisted conception likelihood of detecting heterotopic pregnancy will increased by missed or delayed diagnosis of spontaneous heterotopic pregnancy remains a diagnostic dilemma and a challenge for gynaecologists. In a case series of 13 cases of HP, only one case was a spontaneous $\mathrm{HP}, 6$ with ovulation induction and 6 with $\mathrm{IVF}^{10}$. The mean gestational age at the time of the diagnosis was 8 weeks and $54 \%$ heterotopic pregnancies were detected by TVS. All the patients underwent surgical treatment out of which 10 had laparoscopy and 3 had a laparotomy mainly for significant haemoperitoneum. They found laparoscopy to be useful for the early diagnosis of HP and resulted in good surgical outcomes.

\section{CONCLUSION}

Women with previous ectopic pregnancy, tubal surgery or previous pelvic inflammatory disease, may be at a higher risk and should be scanned at an early gestation to confirm the location of pregnancy. Also a high index of suspicion is necessary in the low risk symptomatic patient with abdominal or pelvic pain in which ultrasound findings are consistent with intrauterine gestation sac while free fluid is noted in the pelvis with or without an adnexal mass.

\section{DISCLOSURE}

All the authors declared no competing interest. 


\section{REFERENCES}

1. De Voe RW, Pratt JH: Simultaneous intrauterine and extrauterine pregnancy. Am j Obstet Gynaecol. 1948; 56:1119-1126.

2. LYnos EA, Levis CS: Diagnostic ultrasound, Rumack CM, Wilson SR, Charboneau JW, editors. 2009; 3(2): 1103.

3. Glassner MJ, Aron E, Eskin BA: Ovulation induction with clomiphene and the rise in heterotopic pregnancies, J Repord Med. 1990 35:175-178.

4. Ahmed A, Tom B, Calabrese P: Ectopic pregnancy diagnosis and the pseudo-sac. Fertil steril. 2004; 81(5):1225-1228.

5. Govindaranjan MJ, Rajan R: Heterotopic pregnancy in natural conception. J Hum Reprod Sci I. 1997; (1):37-38.

6. Peleg D, Bar-Hava, Neaman-Leaven M, Ashkena, Ben-RafaelzJ: Early diagnosis and successful non surgical treatment of viable combined intrauterine and cervical pregnancy. Fertil. Steril. 1994; 62:405.

7. Raziel A, friedler S, Herman A, Strassburger D, Maymon R, Ron-el R: Recurrent heterotopic pregnancy after repeated in-vitro fertilization treatment. Hum rep. 1997; 12:1810-1812.

8. Jerrad D, Tso E, salik R, barish RA: Unsuspected heterotopic pregnancy in a woman without risk factors. Am J Emerg Med. 1992; 10:58-60.

9. Sohail S: Haemorrhagic corpus luteum mimicking heterotopic pregnancy. J Coll Phys Surg Pak. 2005; 15:180-181.

10. Louis- sylvestre, Morice P, Chapron C, Dubuisson : The role of laparoscopy in the diagnosis and management of heterotopic pregnancy. Hum Rep. 1997; 12(5):1100-1102.

11. Jibodu OA, Darne FJ: Spontaneous heterotopic pregnancy presenting withv tubal rupture. Hum Rep. 1997; 12(5):1098-1099.

12. O. Dundar, L Tutuncu, E. Mungen, M. Muhcu, and Y. Z. Yergok: Heterotopic pregnancy: tubal ectopic pregnancy and monochorionic mono-amniotic twin pregnancy: a case report, Perinatal journal. 2006; 14:96-100.

13. H. Fernandez and A. Gervaise, Ectopic pregnancies after infertility treatment: modern diagnosis and therapeutic strategy. Human Reproduction update. 2004; 10(6):503-513.

14. H. M. Xiao, F.Gong, Z. H. Mao, H. Zhang, and G. X. Lu, Analysis of 92 ectopic pregnancy patients after in vitro fertilization and embryo transfer. Journal of Central South University. 2006; 31(4):584-587. 